\title{
Common tripled fixed point theorem in two rectangular b-metric spaces and applications
}

Feng Gu*, Liya Liu

Institute of Applied Mathematics and Department of Mathematics, Hangzhou Normal University, Hangzhou, Zhejiang 310036, China.

Communicated by C. Alaca

\begin{abstract}
In this paper, we establish some new common tripled fixed point theorems for mappings defined on a set equipped with two rectangular b-metrics. We also provide illustrative examples in support of our new results. In the end of the paper, we give an existence and uniqueness theorem for a class of nonlinear integral equations by using the obtained result. The results presented in this paper generalize the well-known comparable results in the literature. (c)2017 All rights reserved.
\end{abstract}

Keywords: Rectangular b-metric space, contractive mappings, tripled coincidence point, common tripled fixed point, $\omega$-compatible mapping pairs. 2010 MSC: 47H10, 54H25.

\section{Introduction and preliminaries}

The concept of b-metric space was introduced by Czerwik [10] which is defined as:

Definition 1.1 ([10]). Let $X$ be a nonempty set and $s \geqslant 1$ be a given real number. A function $d: X \times X \rightarrow$ $[0, \infty)$ is a $b$-metric on $X$, if for all $x, y, z \in X$, the following conditions hold:

(bM1) $d(x, y)=0$ if and only if $x=y$;

(bM2) $d(x, y)=d(y, x)$;

(bM3) $d(x, z) \leqslant s[d(x, y)+d(y, z)]$.

In this case, the pair $(X, d)$ is called a b-metric space, and the number $s$ is called the coefficient of $(X, d)$.

As an important generalizations of usual metric spaces, Branciari [8] introduced the concept of rectangular metric space as follows:

Definition 1.2 ([8]). Let $X$ be a nonempty set, and let $d: X \times X \rightarrow[0, \infty)$ be a mapping such that for all $x, y \in X$, the following conditions hold:

\footnotetext{
*Corresponding author

Email addresses: mathgufeng@163.com (Feng Gu), 846883245@qq.com (Liya Liu)
} 
(RM1) $\mathrm{d}(x, y)=0$ if and only if $x=y$;

(RM2) $d(x, y)=d(y, x)$;

(RM3) $d(x, z) \leqslant d(x, u)+d(u, v)+d(v, z)$ for all $u, v \in X \backslash\{x, y\}$ and $u \neq v$.

Then $(X, d)$ is called a rectangular or generalized metric space.

After that, fixed point results in rectangular metric space have been studied by many authors (see e.g. $[1-3,6,7,9,11,12,15,16,18-25,27,28,30])$.

Recently, George et al. [17] and Roshan et al. [26] introduced the notion of rectangular b-metric space as follows:

Definition $1.3([17,26])$. Let $X$ be a nonempty set, $s \geqslant 1$ be a given real number and let $d: X \times X \rightarrow[0, \infty)$ be a mapping such that for all $x, y \in X$, the following conditions hold:

(RbM1) $d(x, y)=0$ if and only if $x=y$;

(RbM2) $d(x, y)=d(y, x)$;

(RbM3) $d(x, z) \leqslant s[d(x, u)+d(u, v)+d(v, z)]$ for all $u, v \in X \backslash\{x, y\}$ and $u \neq v$.

Then $(X, d)$ is called a rectangular $b$-metric space or a generalized b-metric space, and the number $s$ is called the coefficient of $(X, d)$.

Remark 1.4 ([14]). Every metric space is a rectangular metric space and every rectangular metric space is a rectangular $b$-metric space (with coefficient $s=1$ ). However the converse is not necessarily true ([17, Examples 1.4. and 1.5.]). Also, every metric space is a b-metric space and every b-metric space with coefficient $s$ is a rectangular b-metric space with coefficient $s^{2}$ but the converse is not necessarily true ([17, Examples 1.7]).

Very recently, Ding et al. [13, 14] and Aydi et al. [5] also discussed the fixed point and common fixed point problems of different contractive mapping for rectangular b-metric spaces. However, so far, no one discussed tripled fixed point problem in rectangular b-metric space.

The purpose of this paper is to prove some new common tripled fixed point theorems for mappings defined on a set equipped with two rectangular b-metrics. We also provide an existence and uniqueness theorem of solution for a class of nonlinear integral equations by using the obtained result.

Now, we give some basic notions before introducing some main results.

Definition 1.5 ([17]). Let $(X, d)$ be a rectangular b-metric space, $\left\{x_{n}\right\}$ be a sequence in $X$ and $x \in X$. Then

(a) The sequence $\left\{x_{n}\right\}$ is said to be convergent in $X$ and converges to $x$, if for every $\epsilon>0$ there exists $n_{0} \in \mathbb{N}$ such that $d\left(x_{n}, x\right)<\epsilon$ for all $n>n_{0}$ and this fact is represented by $\lim _{n \rightarrow \infty} x_{n}=x$ or $x_{n} \rightarrow x$ as $n \rightarrow \infty$.

(b) The sequence $\left\{x_{n}\right\}$ is said to be Cauchy sequence in $(X, d)$ if for every $\epsilon>0$ there exists $n_{0} \in \mathbb{N}$ such that $d\left(x_{n}, x_{n+p}\right)<\epsilon$ for all $n>n_{0}, p>0$ or equivalently, if $\lim _{n \rightarrow \infty} d\left(x_{n}, x_{n+p}\right)=0$ for all $p>0$.

(c) $(X, d)$ is said to be a complete rectangular b-metric space if every Cauchy sequence in $X$ converges to some $x \in X$.

Note that limit of sequence in a rectangular b-metric space is not necessarily unique and also every convergent sequence in a rectangular b-metric space is not necessarily Cauchy sequence ([17, Examples 1.7]).

Definition 1.6 ([29]). An element $(x, y, z) \in X \times X \times X$ is called a tripled fixed point of the mapping $F: X \times X \times X \rightarrow X$, if $F(x, y, z)=x, F(y, z, x)=y$ and $F(z, x, y)=z$. 
Definition 1.7 ([4]). An element $(x, y, z) \in X \times X \times X$ is called a tripled coincidence point of mappings $F: X \times X \times X \rightarrow X$ and $g: X \rightarrow X$, if $F(x, y, z)=g x, F(y, z, x)=g y$ and $F(z, x, y)=g z$. In this case, $(g x, g y, g z)$ is called a triple point of coincidence of the mappings $g$ and $F$.

Definition $1.8([4])$. An element $(x, y, z) \in X \times X \times X$ is called a common tripled fixed point of mappings $F: X \times X \times X \rightarrow X$ and $g: X \rightarrow X$, if $F(x, y, z)=g x=x, F(y, z, x)=g y=y$ and $F(z, x, y)=g z=z$.

Definition 1.9 ([4]). Let $X$ be a nonempty set. A pair of mappings $F: X \times X \times X \rightarrow X$ and $g: X \rightarrow X$ are called to be $w$-compatible, if $g F(x, y, z)=F(g x, g y, g z)$ whenever $F(x, y, z)=g x$ and $F(y, z, x)=g y$.

\section{Main results}

Theorem 2.1. Let $d_{1}$ and $d_{2}$ be two rectangular b-metrics on $X$ such that $d_{2}(x, y) \leqslant d_{1}(x, y)$ for all $x, y \in X$, $\left(\mathrm{X}, \mathrm{d}_{1}\right)$ with coefficient $\mathrm{s} \geqslant 1$, and $\mathrm{F}: \mathrm{X} \times \mathrm{X} \times \mathrm{X} \rightarrow \mathrm{X}$ and $\mathrm{g}: \mathrm{X} \rightarrow \mathrm{X}$ be two mappings. Suppose that there exist $k_{1}, k_{2}$ and $k_{3}$ in $[0,1)$ with $0 \leqslant k_{1}+k_{2}+k_{3}<1$ and $0 \leqslant s k_{3}<1$ such that the condition

$$
\begin{aligned}
& d_{1}(F(x, y, z), F(u, v, w))+d_{1}(F(y, z, x), F(v, w, u))+d_{1}(F(z, x, y), F(w, u, v)) \\
& \leqslant k_{1}\left[d_{2}(g x, g u)+d_{2}(g y, g v)+d_{2}(g z, g w)\right] \\
& +k_{2}\left[d_{2}(g x, F(x, y, z))+d_{2}(g y, F(y, z, x))+d_{2}(g z, F(z, x, y))\right] \\
& +k_{3}\left[d_{2}(g u, F(u, v, w))+d_{2}(g v, F(v, w, u))+d_{2}(g w, F(w, u, v))\right],
\end{aligned}
$$

holds for all $(x, y, z),(u, v, w) \in X \times X \times X$.

If $\mathrm{F}(\mathrm{X} \times \mathrm{X} \times \mathrm{X}) \subseteq \mathrm{g}(\mathrm{X})$ and $\mathrm{g}(\mathrm{X})$ is $\mathrm{d}_{1}$-complete, then $\mathrm{g}$ and $\mathrm{F}$ have a tripled coincidence point $(x, y, z) \in$ $\mathrm{X} \times \mathrm{X} \times \mathrm{X}$, satisfying that $\mathrm{gx}=\mathrm{F}(\mathrm{x}, \mathrm{y}, z)=\mathrm{gy}=\mathrm{F}(\mathrm{y}, z, \mathrm{x})=\mathrm{g} z=\mathrm{F}(z, \mathrm{x}, \mathrm{y})$.

Moreover, if $\mathrm{g}$ and $\mathrm{F}$ are $\mathrm{w}$-compatible, then $\mathrm{g}$ and $\mathrm{F}$ have a unique common tripled fixed point of the form $(\mathrm{u}, \mathrm{u}, \mathrm{u})$, which satisfies that $\mathrm{u}=\mathrm{gu}=\mathrm{F}(\mathrm{u}, \mathrm{u}, \mathrm{u})$.

Proof. Let $\left(x_{0}, y_{0}, z_{0}\right) \in X \times X \times X$, by making the use of $F(X \times X \times X) \subseteq g(X)$, then there exist $x_{1}, y_{1}, z_{1} \in X$ such that $g x_{1}=F\left(x_{0}, y_{0}, z_{0}\right), g y_{1}=F\left(y_{0}, z_{0}, x_{0}\right)$ and $g z_{1}=F\left(z_{0}, x_{0}, y_{0}\right)$. By similar arguments as above we can show that there exist $x_{2}, y_{2}, z_{2} \in X$ such that $g x_{2}=T\left(x_{1}, y_{1}, z_{1}\right), g y_{2}=T\left(y_{1}, z_{1}, x_{1}\right), g z_{2}=$ $\mathrm{T}\left(z_{1}, x_{1}, y_{1}\right), \cdots$. Repeating the above procedure, we can construct three sequences $\left\{x_{n}\right\},\left\{y_{n}\right\}$ and $\left\{z_{n}\right\}$ such that

$$
g x_{n+1}=F\left(x_{n}, y_{n}, z_{n}\right), \quad g y_{n+1}=F\left(y_{n}, z_{n}, x_{n}\right), \quad g z_{n+1}=F\left(z_{n}, x_{n}, y_{n}\right), \quad \forall n \geqslant 0 .
$$

Without loss of generality, we can assume that $g x_{n} \neq g x_{n+1}, g y_{n} \neq g y_{n+1}$ and $g z_{n} \neq g z_{n+1}$, for all $n \geqslant 0$.

By taking $(x, y, z)=\left(x_{n}, y_{n}, z_{n}\right)$ and $(u, v, w)=\left(x_{n+1}, y_{n+1}, z_{n+1}\right)$ in (2.1), we obtain

$$
\begin{aligned}
& d_{1}\left(g x_{n+1}, g x_{n+2}\right)+d_{1}\left(g y_{n+1}, g y_{n+2}\right)+d_{1}\left(g z_{n+1}, g z_{n+2}\right) \\
&= d_{1}\left(F\left(x_{n}, y_{n}, z_{n}\right), F\left(x_{n+1}, y_{n+1}, z_{n+1}\right)\right)+d_{1}\left(F\left(y_{n}, z_{n}, x_{n}\right), F\left(y_{n+1}, z_{n+1}, x_{n+1}\right)\right) \\
&+d_{1}\left(F\left(z_{n}, x_{n}, y_{n}\right), F\left(z_{n+1}, x_{n+1}, y_{n+1}\right)\right) \\
& \leqslant k_{1}\left[d_{2}\left(g x_{n}, g x_{n+1}\right)+d_{2}\left(g y_{n}, g y_{n+1}\right)+d_{2}\left(g z_{n}, g z_{n+1}\right)\right] \\
&+k_{2}\left[d_{2}\left(g x_{n}, F\left(x_{n}, y_{n}, z_{n}\right)\right)+d_{2}\left(g y_{n}, F\left(y_{n}, z_{n}, x_{n}\right)\right)+d_{2}\left(g z_{n}, F\left(z_{n}, x_{n}, y_{n}\right)\right)\right] \\
&+k_{3}\left[d_{2}\left(g x_{n+1}, F\left(x_{n+1}, y_{n+1}, z_{n+1}\right)\right)\right. \\
&\left.+d_{2}\left(g y_{n+1}, F\left(y_{n+1}, z_{n+1}, x_{n+1}\right)\right)+d_{2}\left(g z_{n+1}, F\left(z_{n+1}, x_{n+1}, y_{n+1}\right)\right)\right] \\
&= k_{1}\left[d_{2}\left(g x_{n}, g x_{n+1}\right)+d_{2}\left(g y_{n}, g y_{n+1}\right)+d_{2}\left(g z_{n}, g z_{n+1}\right)\right] \\
&+k_{2}\left[d_{2}\left(g x_{n}, g x_{n+1}\right)+d_{2}\left(g y_{n}, g y_{n+1}\right)+d_{2}\left(g z_{n}, g z_{n+1}\right)\right] \\
&+k_{3}\left[d_{2}\left(g x_{n+1}, g x_{n+2}\right)+d_{2}\left(g y_{n+1}, g y_{n+2}\right)+d_{2}\left(g z_{n+1}, g z_{n+2}\right)\right] \\
& \leqslant k_{1}\left[d_{1}\left(g x_{n}, g x_{n+1}\right)+d_{1}\left(g y_{n}, g y_{n+1}\right)+d_{1}\left(g z_{n}, g z_{n+1}\right)\right]
\end{aligned}
$$




$$
\begin{aligned}
& +k_{2}\left[d_{1}\left(g x_{n}, g x_{n+1}\right)+d_{1}\left(g y_{n}, g y_{n+1}\right)+d_{1}\left(g z_{n}, g z_{n+1}\right)\right] \\
& +k_{3}\left[d_{1}\left(g x_{n+1}, g x_{n+2}\right)+d_{1}\left(g y_{n+1}, g y_{n+2}\right)+d_{1}\left(g z_{n+1}, g z_{n+2}\right)\right] .
\end{aligned}
$$

It follows from (2.2) that

$$
\begin{aligned}
d_{1}\left(g x_{n+1}, g x_{n+2}\right)+ & d_{1}\left(g y_{n+1}, g y_{n+2}\right)+d_{1}\left(g z_{n+1}, g z_{n+2}\right) \\
& \leqslant\left(\frac{k_{1}+k_{2}}{1-k_{3}}\right)\left[d_{1}\left(g x_{n}, g x_{n+1}\right)+d_{1}\left(g y_{n}, g y_{n+1}\right)+d_{1}\left(g z_{n}, g z_{n+1}\right)\right] .
\end{aligned}
$$

Taking $k=\frac{k_{1}+k_{2}}{1-k_{3}}$, by the condition $0 \leqslant k_{1}+k_{2}+k_{3}<1$, then we have $0 \leqslant k<1$. (2.3) implies that

$$
\begin{aligned}
d_{1}\left(g x_{n+1}, g x_{n+2}\right)+ & d_{1}\left(g y_{n+1}, g y_{n+2}\right)+d_{1}\left(g z_{n+1}, g z_{n+2}\right) \\
& \leqslant k\left[d_{1}\left(g x_{n}, g x_{n+1}\right)+d_{1}\left(g y_{n}, g y_{n+1}\right)+d_{1}\left(g z_{n}, g z_{n+1}\right)\right] .
\end{aligned}
$$

By taking $\delta_{n}=d_{1}\left(g x_{n}, g x_{n+1}\right)+d_{1}\left(g y_{n}, g y_{n+1}\right)+d_{1}\left(g z_{n}, g z_{n+1}\right)$. Repeating the above inequality (2.4) $n+1$ times, we obtain,

$$
\delta_{n+1} \leqslant k \delta_{n} \leqslant k^{2} \delta_{n-1} \leqslant \cdots \leqslant k^{n+1} \delta_{0} .
$$

As $(x, y, z)=\left(x_{n}, y_{n}, z_{n}\right)$ and $(u, v, w)=\left(x_{n+2}, y_{n+2}, z_{n+2}\right)$ in (2.1), also with (2.5), we get

$$
\begin{aligned}
d_{1}\left(g x_{n+1}, g x_{n+3}\right)+ & \left.d_{1}\left(g y_{n+1}, g y_{n+3}\right)\right)+d_{1}\left(g z_{n+1}, g z_{n+3}\right) \\
= & d_{1}\left(F\left(x_{n}, y_{n}, z_{n}\right), F\left(x_{n+2}, y_{n+2}, z_{n+2}\right)\right)+d_{1}\left(F\left(y_{n}, z_{n}, x_{n}\right), F\left(y_{n+2}, z_{n+2}, x_{n+2}\right)\right) \\
& +d_{1}\left(F\left(z_{n}, x_{n}, y_{n}\right), F\left(z_{n+2}, x_{n+2}, y_{n+2}\right)\right) \\
\leqslant & k_{1}\left[d_{2}\left(g x_{n}, g x_{n+2}\right)+d_{2}\left(g y_{n}, g y_{n+2}\right)+d_{2}\left(g z_{n}, g z_{n+2}\right)\right] \\
& +k_{2}\left[d_{2}\left(g x_{n}, F\left(x_{n}, y_{n}, z_{n}\right)\right)+d_{2}\left(g y_{n}, F\left(y_{n}, z_{n}, x_{n}\right)\right)+d_{2}\left(g z_{n}, F\left(z_{n}, x_{n}, y_{n}\right)\right)\right] \\
& +k_{3}\left[d_{2}\left(g x_{n+2}, F\left(x_{n+2}, y_{n+2}, z_{n+2}\right)\right)\right. \\
& \left.+d_{2}\left(g y_{n+2}, F\left(y_{n+2}, z_{n+2}, x_{n+2}\right)\right)+d_{2}\left(g z_{n+2}, F\left(z_{n+2}, x_{n+2}, y_{n+2}\right)\right)\right] \\
= & k_{1}\left[d_{2}\left(g x_{n}, g x_{n+2}\right)+d_{2}\left(g y_{n}, g y_{n+2}\right)+d_{2}\left(g z_{n}, g z_{n+2}\right)\right] \\
& +k_{2}\left[d_{2}\left(g x_{n}, g x_{n+1}\right)+d_{2}\left(g y_{n}, g y_{n+1}\right)+d_{2}\left(g z_{n}, g z_{n+1}\right)\right] \\
& +k_{3}\left[d_{2}\left(g x_{n+2}, g x_{n+3}\right)+d_{2}\left(g y_{n+2}, g y_{n+3}\right)+d_{2}\left(g z_{n+2}, g z_{n+3}\right)\right] \\
\leqslant & k_{1}\left[d_{2}\left(g x_{n}, g x_{n+2}\right)+d_{2}\left(g y_{n}, g y_{n+2}\right)+d_{2}\left(g z_{n}, g z_{n+2}\right)\right] \\
& +k_{2}\left[d_{2}\left(g x_{n}, g x_{n+1}\right)+d_{2}\left(g y_{n}, g y_{n+1}\right)+d_{2}\left(g z_{n}, g z_{n+1}\right)\right] \\
& +k_{3} k^{2}\left[d_{2}\left(g x_{n}, g x_{n+1}\right)+d_{2}\left(g y_{n}, g y_{n+1}\right)+d_{2}\left(g z_{n}, g z_{n+1}\right)\right] \\
= & k_{1}\left[d_{2}\left(g x_{n}, g x_{n+2}\right)+d_{2}\left(g y_{n}, g y_{n+2}\right)+d_{2}\left(g z_{n}, g z_{n+2}\right)\right] \\
& +\left(k_{2}+k_{3} k^{2}\right)\left[d_{2}\left(g x_{n}, g x_{n+1}\right)+d_{2}\left(g y_{n}, g y_{n+1}\right)+d_{2}\left(g z_{n}, g z_{n+1}\right)\right] \\
\leqslant & k_{1}\left[d_{1}\left(g x_{n}, g x_{n+2}\right)+d_{1}\left(g y_{n}, g y_{n+2}\right)+d_{1}\left(g z_{n}, g z_{n+2}\right)\right] \\
& +\left(k_{2}+k_{3} k^{2}\right)\left[d_{1}\left(g x_{n}, g x_{n+1}\right)+d_{1}\left(g y_{n}, g y_{n+1}\right)+d_{1}\left(g z_{n}, g z_{n+1}\right)\right]
\end{aligned}
$$

By taking

$$
\delta_{n}^{*}=d_{1}\left(g x_{n}, g x_{n+2}\right)+d_{1}\left(g y_{n}, g y_{n+2}\right)+d_{1}\left(g z_{n}, g z_{n+2}\right),
$$

from $k=\frac{k_{1}+k_{2}}{1-k_{3}} \in[0,1)$ we have

$$
k_{1}+k_{2}+k_{3} k^{2} \leqslant k_{1}+k_{2}+k_{3} k=k_{1}+k_{2}+k_{3} \frac{k_{1}+k_{2}}{1-k_{3}}=\frac{k_{1}+k_{2}}{1-k_{3}}=k .
$$

Consequently, by the use of (2.5) and (2.6), we have

$$
\delta_{n+1}^{*} \leqslant k_{1} \delta_{n}^{*}+\left(k_{2}+k_{3} k^{2}\right) \delta_{n} \leqslant\left(k_{1}+k_{2}+k_{3} k^{2}\right) \max \left\{\delta_{n}, \delta_{n}^{*}\right\} \leqslant k \max \left\{\delta_{n}, \delta_{n}^{*}\right\} .
$$


It follows from (2.5) and (2.7) that

$$
\begin{aligned}
\delta_{n+1}^{*} & \leqslant k \max \left\{\delta_{n}, \delta_{n}^{*}\right\} \leqslant k \max \left\{k \delta_{n-1}, k \max \left\{\delta_{n-1}, \delta_{n-1}^{*}\right\}\right\}=k^{2} \max \left\{\delta_{n-1}, \delta_{n-1}^{*}\right\} \\
& \leqslant k^{3} \max \left\{\delta_{n-2}, \delta_{n-2}^{*}\right\} \leqslant \cdots \leqslant k^{n+1} \max \left\{\delta_{0}, \delta_{0}^{*}\right\} .
\end{aligned}
$$

Next, we show that $\left\{g x_{n}\right\},\left\{g y_{n}\right\}$ and $\left\{g z_{n}\right\}$ are Cauchy sequences in $g(X)$. For this, we consider $\mathrm{d}_{1}\left(x_{n}, x_{n+p}\right)$ in two cases.

Case 1. $p$ is an odd number, assume that $p=2 m+1$, then using (RbM3) we obtain

$$
\begin{aligned}
d_{1}\left(g x_{n}, g x_{n+p}\right)= & d_{1}\left(g x_{n}, g x_{n+2 m+1}\right) \\
\leqslant & s\left[d_{1}\left(g x_{n}, g x_{n+1}\right)+d_{1}\left(g x_{n+1}, g x_{n+2}\right)+d_{1}\left(g x_{n+2}, g x_{n+2 m+1}\right)\right] \\
\leqslant & s\left[d_{1}\left(g x_{n}, g x_{n+1}\right)+d_{1}\left(g x_{n+1}, g x_{n+2}\right)\right] \\
& +s^{2}\left[d_{1}\left(g x_{n+2}, g x_{n+3}\right)+d_{1}\left(g x_{n+3}, g x_{n+4}\right)+d_{1}\left(g x_{n+4}, g x_{n+2 m+1}\right)\right] \\
\leqslant & s\left[d_{1}\left(g x_{n}, g x_{n+1}\right)+d_{1}\left(g x_{n+1}, g x_{n+2}\right)\right]+s^{2}\left[d_{1}\left(g x_{n+2}, g x_{n+3}\right)+d_{1}\left(g x_{n+3}, g x_{n+4}\right)\right] \\
& +s^{3}\left[d_{1}\left(g x_{n+4}, g x_{n+5}\right)+d_{1}\left(g x_{n+5}, g x_{n+6}\right)+d_{1}\left(g x_{n+6}, g x_{n+2 m+1}\right)\right] \\
\leqslant & \cdots \\
\leqslant & s\left[d_{1}\left(g x_{n}, g x_{n+1}\right)+d_{1}\left(g x_{n+1}, g x_{n+2}\right)\right]+s^{2}\left[d_{1}\left(g x_{n+2}, g x_{n+3}\right)+d_{1}\left(g x_{n+3}, g x_{n+4}\right)\right] \\
& +s^{3}\left[d_{1}\left(g x_{n+4}, g x_{n+5}\right)+d_{1}\left(g x_{n+5}, g x_{n+6}\right)\right]+\cdots \\
& +s^{m}\left[d_{1}\left(g x_{n+2 m-2}, g x_{n+2 m-1}\right)+d_{1}\left(g x_{n+2 m-1}, g x_{n+2 m}\right)+d_{1}\left(g x_{n+2 m}, g x_{n+2 m+1}\right)\right] .
\end{aligned}
$$

That is

$$
\begin{aligned}
d_{1}\left(g x_{n}, g x_{n+p}\right)= & d_{1}\left(g x_{n}, g x_{n+2 m+1}\right) \\
\leqslant & s\left[d_{1}\left(g x_{n}, g x_{n+1}\right)+d_{1}\left(g x_{n+1}, g x_{n+2}\right)\right]+s^{2}\left[d_{1}\left(g x_{n+2}, g x_{n+3}\right)+d_{1}\left(g x_{n+3}, g x_{n+4}\right)\right] \\
& +s^{3}\left[d_{1}\left(g x_{n+4}, g x_{n+5}\right)+d_{1}\left(g x_{n+5}, g x_{n+6}\right)\right]+\cdots \\
& +s^{m}\left[d_{1}\left(g x_{n+2 m-2}, g x_{n+2 m-1}\right)+d_{1}\left(g x_{n+2 m-1}, g x_{n+2 m}\right)\right]+s^{m} d_{1}\left(g x_{n+2 m}, g x_{n+2 m+1}\right) .
\end{aligned}
$$

We can similarly prove the following result

$$
\begin{aligned}
d_{1}\left(g y_{n}, g y_{n+p}\right)= & d_{1}\left(g y_{n}, g y_{n+2 m+1}\right) \\
\leqslant & s\left[d_{1}\left(g y_{n}, g y_{n+1}\right)+d_{1}\left(g y_{n+1}, g y_{n+2}\right)\right]+s^{2}\left[d_{1}\left(g y_{n+2}, g y_{n+3}\right)+d_{1}\left(g y_{n+3}, g y_{n+4}\right)\right] \\
& +s^{3}\left[d_{1}\left(g y_{n+4}, g y_{n+5}\right)+d_{1}\left(g y_{n+5}, g y_{n+6}\right)\right]+\cdots \\
& +s^{m}\left[d_{1}\left(g y_{n+2 m-2}, g y_{n+2 m-1}\right)+d_{1}\left(g y_{n+2 m-1}, g y_{n+2 m}\right)\right]+s^{m} d_{1}\left(g y_{n+2 m}, g y_{n+2 m+1}\right),
\end{aligned}
$$

and

$$
\begin{aligned}
d_{1}\left(g z_{\mathfrak{n}}, g z_{\mathfrak{n}+p}\right)= & d_{1}\left(g z_{\mathfrak{n}}, g z_{\mathfrak{n}+2 m+1}\right) \\
\leqslant & s\left[d_{1}\left(g z_{\mathfrak{n}}, g z_{\mathfrak{n}+1}\right)+d_{1}\left(g z_{\mathfrak{n}+1}, g z_{\mathfrak{n}+2}\right)\right]+s^{2}\left[d_{1}\left(g z_{\mathfrak{n}+2}, g z_{\mathfrak{n}+3}\right)+d_{1}\left(g z_{\mathfrak{n}+3}, g z_{\mathfrak{n}+4}\right)\right] \\
& +s^{3}\left[d_{1}\left(g z_{\mathfrak{n}+4}, g z_{\mathfrak{n}+5}\right)+d_{1}\left(g z_{\mathfrak{n}+5}, g z_{\mathfrak{n}+6}\right)\right]+\cdots \\
& +s^{m}\left[d_{1}\left(g z_{\mathfrak{n}+2 m-2}, g z_{\mathfrak{n}+2 m-1}\right)+d_{1}\left(g z_{\mathfrak{n}+2 m-1}, g z_{\mathfrak{n}+2 m}\right)\right]+s^{m} d_{1}\left(g z_{\mathfrak{n}+2 m}, g z_{\mathfrak{n}+2 m+1}\right) .
\end{aligned}
$$

Combining (2.5), (2.9), (2.10) and (2.11), we have

$$
\begin{aligned}
d_{1}\left(g x_{n}, g x_{n+p}\right)+ & d_{1}\left(g y_{n}, g y_{n+p}\right)+d_{1}\left(g z_{n}, g z_{n+p}\right) \\
& =d_{1}\left(g x_{n}, g x_{n+2 m+1}\right)+d_{1}\left(g y_{n}, g y_{n+2 m+1}\right)+d_{1}\left(g z_{n}, g z_{n+2 m+1}\right) \\
& \leqslant s\left(\delta_{n}+\delta_{n+1}\right)+s^{2}\left(\delta_{n+2}+\delta_{n+3}\right)+\cdots+s^{m}\left(\delta_{n+2 m-2}+\delta_{n+2 m-1}\right)+s^{m} \delta_{n+2 m} \\
& \leqslant s\left(k^{n}+k^{n+1}\right) \delta_{0}+s^{2}\left(k^{n+2}+k^{n+3}\right) \delta_{0}+\cdots+s^{m}\left(k^{n+2 m-2}+k^{n+2 m-1}\right) \delta_{0}+s^{m} k^{n+2 m} \delta_{0} \\
& =s k^{n}(1+k)\left[1+s k^{2}+\left(s k^{2}\right)^{2}+\cdots+\left(s k^{2}\right)^{m-1}\right] \delta_{0}+s^{m} k^{n+2 m} \delta_{0}
\end{aligned}
$$




$$
\begin{aligned}
& = \begin{cases}{\left[s k^{n}(1+k) \cdot m+s^{m} k^{n+2 m}\right] \delta_{0},} & s k^{2}=1, \\
\left(s k^{n}(1+k) \cdot \frac{1-\left(s k^{2}\right)^{m}}{1-s k^{2}}+s^{m} k^{n+2 m}\right) \delta_{0}, & s k^{2} \neq 1,\end{cases} \\
& \leqslant \begin{cases}{\left[s k^{n}(1+k) \cdot m+s^{m} k^{n+2 m}\right] \delta_{0},} & s k^{2}=1, \\
\left(\frac{s k^{n}(1+k)}{1-s k^{2}}+s^{m} k^{n+2 m}\right) \delta_{0}, & s k^{2} \neq 1 .\end{cases}
\end{aligned}
$$

Case 2. $p$ is an even number, assume that $p=2 m$, then using ( $R b M 3)$ we obtain

$$
\begin{aligned}
d_{1}\left(g x_{n}, g x_{n+p}\right)= & d_{1}\left(g x_{n}, g x_{n+2 m}\right) \\
\leqslant & s\left[d_{1}\left(g x_{n}, g x_{n+1}\right)+d_{1}\left(g x_{n+1}, g x_{n+2}\right)+d_{1}\left(g x_{n+2}, g x_{n+2 m}\right)\right] \\
\leqslant & s\left[d_{1}\left(g x_{n}, g x_{n+1}\right)+d_{1}\left(g x_{n+1}, g x_{n+2}\right)\right] \\
& +s^{2}\left[d_{1}\left(g x_{n+2}, g x_{n+3}\right)+d_{1}\left(g x_{n+3}, g x_{n+4}\right)+d_{1}\left(g x_{n+4}, g x_{n+2 m}\right)\right] \\
\leqslant & \cdots \\
\leqslant & s\left[d_{1}\left(g x_{n}, g x_{n+1}\right)+d_{1}\left(g x_{n+1}, g x_{n+2}\right)\right]+s^{2}\left[d_{1}\left(g x_{n+2}, g x_{n+3}\right)+d_{1}\left(g x_{n+3}, g x_{n+4}\right)\right] \\
& +\cdots+s^{m-1}\left[d_{1}\left(g x_{n+2 m-4}, g x_{n+2 m-3}\right)+d_{1}\left(g x_{n+2 m-3}, g x_{n+2 m-2}\right)\right] \\
& +s^{m-1} d_{1}\left(g x_{n+2 m-2}, g x_{n+2 m}\right) .
\end{aligned}
$$

By similar arguments as above,

$$
\begin{aligned}
d_{1}\left(g y_{n}, g y_{n+p}\right)= & d_{1}\left(g y_{n}, g y_{n+2 m}\right) \\
\leqslant & s\left[d_{1}\left(g y_{n}, g y_{n+1}\right)+d_{1}\left(g y_{n+1}, g y_{n+2}\right)\right]+s^{2}\left[d_{1}\left(g y_{n+2}, g y_{n+3}\right)+d_{1}\left(g y_{n+3}, g y_{n+4}\right)\right] \\
& +\cdots+s^{m-1}\left[d_{1}\left(g y_{n+2 m-4}, g y_{n+2 m-3}\right)+d_{1}\left(g y_{n+2 m-3}, g x_{n+2 m-2}\right)\right] \\
& +s^{m-1}\left[d_{1}\left(g y_{n+2 m-2}, g y_{n+2 m}\right)\right]
\end{aligned}
$$

and

$$
\begin{aligned}
d_{1}\left(g z_{n}, g z_{n+p}\right)= & d_{1}\left(g z_{n}, g z_{n+2 m}\right) \\
\leqslant & s\left[d_{1}\left(g z_{n}, g z_{n+1}\right)+d_{1}\left(g z_{n+1}, g z_{n+2}\right)\right]+s^{2}\left[d_{1}\left(g z_{n+2}, g z_{n+3}\right)+d_{1}\left(g z_{n+3}, g z_{n+4}\right)\right] \\
& +\cdots+s^{m-1}\left[d_{1}\left(g z_{n+2 m-4}, g z_{n+2 m-3}\right)\right. \\
& \left.+d_{1}\left(g z_{n+2 m-3}, g x_{n+2 m-2}\right)\right]+s^{m-1}\left[d_{1}\left(g z_{n+2 m-2}, g z_{n+2 m}\right)\right] .
\end{aligned}
$$

Combining (2.5), (2.8), (2.13), (2.14) and (2.15), we have

$$
\begin{aligned}
& d_{1}\left(g x_{n}, g x_{n+p}\right)+d_{1}\left(g y_{n}, g y_{n+p}\right)+d_{1}\left(g z_{n}, g z_{n+p}\right) \\
&= d_{1}\left(g x_{n}, g x_{n+2 m}\right)+d_{1}\left(g y_{n}, g y_{n+2 m}\right)+d_{1}\left(g z_{n}, g z_{n+2 m}\right) \\
& \leqslant s\left(\delta_{n}+\delta_{n+1}\right)+s^{2}\left(\delta_{n+2}+\delta_{n+3}\right)+\cdots+s^{m-1}\left(\delta_{n+2 m-4}+\delta_{n+2 m-3}\right)+s{ }^{m-1} \delta_{n+2 m-2}^{*} \\
& \leqslant s\left(k^{n}+k^{n+1}\right) \delta_{0}+s^{2}\left(k^{n+2}+k^{n+3}\right) \delta_{0} \\
&+\cdots+s^{m-1}\left(k^{n+2 m-4}+k^{n+2 m-3}\right) \delta_{0}+s^{m-1} k^{n+2 m-2} \max \left\{\delta_{0}, d_{0}^{*}\right\} \\
&= s k^{n}(1+k)\left[1+s k^{2}+\left(s k^{2}\right)^{2}+\cdots+\left(s k^{2}\right)^{m-2}\right] \delta_{0}+s^{m-1} k^{n+2 m-2} \max \left\{\delta_{0}, \delta_{0}^{*}\right\} \\
&= \begin{cases}s k^{n}(1+k)(m-1) \delta_{0}+s^{m-1} k^{n+2 m-2} \max \left\{\delta_{0}, \delta_{0}^{*}\right\}, & s k^{2}=1, \\
\left.s k^{n}(1+k) \cdot \frac{1-\left(s k^{2}\right)^{m-1}}{1-s k^{2}} \cdot \delta_{0}+s^{m-1} k^{n+2 m-2} \max _{\{} d_{0}, d_{0}^{*}\right\}, & s k^{2} \neq 1,\end{cases} \\
& \leqslant \begin{cases}s k^{n}(1+k)(m-1) \delta_{0}+s^{m-1} k^{n+2 m-2} \max \left\{\delta_{0}, \delta_{0}^{*}\right\}, & s k^{2}=1, \\
\frac{s k^{n}(1+k)}{1-s k^{2}} \cdot \delta_{0}+s^{m-1} k^{n+2 m-2} \max \left\{\delta_{0}, \delta_{0}^{*}\right\}, & s k^{2} \neq 1 .\end{cases}
\end{aligned}
$$


Since $k \in[0,1)$, so $k^{n} \rightarrow 0$ as $n \rightarrow \infty$. Taking limit as $n \rightarrow \infty$ in (2.12) and (2.16), we get

$$
\lim _{n \rightarrow \infty}\left[d_{1}\left(g x_{n}, g x_{n+p}\right)+d_{1}\left(g y_{n}, g y_{n+p}\right)+d_{1}\left(g z_{n}, g z_{n+p}\right)\right]=0,
$$

which implies that $\left\{g x_{n}\right\},\left\{g y_{n}\right\}$ and $\left\{g z_{n}\right\}$ are Cauchy sequences in $g(X)$. Since $g(X)$ is complete, then there exist $x, y, z \in X$ such that

$$
\lim _{n \rightarrow \infty} g x_{n}=g x, \quad \lim _{n \rightarrow \infty} g y_{n}=g y, \text { and } \lim _{n \rightarrow \infty} g z_{n}=g z .
$$

It follows from (2.1) and (2.5) that

$$
\begin{aligned}
d_{1}\left(g x_{n+1}, F(\right. & x, y, z))+d_{1}\left(g y_{n+1}, F(y, z, x)\right)+d_{1}\left(g z_{n+1}, F(z, x, y)\right) \\
= & d_{1}\left(F\left(x_{n}, y_{n}, z_{n}\right), F(x, y, z)\right)+d_{1}\left(F\left(y_{n}, z_{n}, x_{n}\right), F(y, z, x)\right)+d_{1}\left(F\left(z_{n}, x_{n}, y_{n}\right), F(z, x, y)\right) \\
\leqslant & k_{1}\left[d_{2}\left(g x_{n}, g x\right)+d_{2}\left(g y_{n}, g y\right)+d_{2}\left(g z_{n}, g z\right)\right] \\
& +k_{2}\left[d_{2}\left(g x_{n}, F\left(x_{n}, y_{n}, z_{n}\right)\right)+d_{2}\left(g y_{n}, F\left(y_{n}, x_{n}, z_{n}\right)\right)+d_{2}\left(g z_{n}, F\left(z_{n}, x_{n}, y_{n}\right)\right)\right] \\
& +k_{3}\left[d_{2}(g x, F(x, y, z))+d_{2}(g y, F(y, z, x))+d_{2}(g z, F(z, x, y))\right] \\
= & k_{1}\left[d_{2}\left(g x_{n}, g x\right)+d_{2}\left(g y_{n}, g y\right)+d_{2}\left(g z_{n}, g z\right)\right] \\
& +k_{2}\left[d_{2}\left(g x_{n}, g x_{n+1}\right)+d_{2}\left(g y_{n}, g y_{n+1}\right)+d_{2}\left(g z_{n}, g z_{n+1}\right)\right] \\
& +k_{3}\left[d_{2}(g x, F(x, y, z))+d_{2}(g y, F(y, z, x))+d_{2}(g z, F(z, x, y))\right] \\
\leqslant & k_{1}\left[d_{1}\left(g x_{n}, g x\right)+d_{1}\left(g y_{n}, g y\right)+d_{1}\left(g z_{n}, g z\right)\right] \\
& +k_{2}\left[d_{1}\left(g x_{n}, g x_{n}+1\right)+d_{1}\left(g y_{n}, g y_{n+1}\right)+d_{1}\left(g z_{n}, g z_{n}+1\right)\right] \\
& +k_{3}\left[d_{1}(g x, F(x, y, z))+d_{1}(g y, F(y, z, x))+d_{1}(g z, F(z, x, y))\right] \\
= & k_{1}\left[d_{1}\left(g x_{n}, g x\right)+d_{1}\left(g y_{n}, g y\right)+d_{1}\left(g z_{n}, g z\right)\right]+k_{2} \delta_{n} \\
& +k_{3}\left[d_{1}(g x, F(x, y, z))+d_{1}(g y, F(y, z, x))+d_{1}(g z, F(z, x, y))\right] \\
\leqslant & k_{1}\left[d_{1}\left(g x_{n}, g x\right)+d_{1}\left(g y_{n}, g y\right)+d_{1}\left(g z_{n}, g z\right)\right]+k_{2} k^{n} \delta_{0} \\
& +k_{3}\left[d_{1}(g x, F(x, y, z))+d_{1}(g y, F(y, z, x))+d_{1}(g z, F(z, x, y))\right]
\end{aligned}
$$

Applying (RbM3), (2.17) and (2.5) we have

$$
\begin{aligned}
& d_{1}(g x, F(x, y, z))+d_{1}(g y, F(y, z, x))+d_{1}(g z, F(z, x, y)) \\
& \leqslant s\left[d_{1}\left(g x, g x_{n}\right)+d_{1}\left(g x_{n}, g x_{n+1}\right)+d_{1}\left(g x_{n+1}, F(x, y, z)\right)\right] \\
&+s\left[d_{1}\left(g y, g y_{n}\right)+d_{1}\left(g y_{n}, g y_{n+1}\right)+d_{1}\left(g y_{n+1}, F(y, z, x)\right)\right] \\
&+s\left[d_{1}\left(g z, g z_{n}\right)+d_{1}\left(g z_{n}, g z_{n+1}\right)+d_{1}\left(g z_{n+1}, F(z, x, y)\right)\right] \\
&= s\left[d_{1}\left(g x, g x_{n}\right)+d_{1}\left(g y, g y_{n}\right)+d_{1}\left(g z, g z_{n}\right)\right]+s \delta_{n} \\
&+s\left[d_{1}\left(g x_{n+1}, F(x, y, z)\right)+d_{1}\left(g y_{n+1}, F(y, z, x)\right)+d_{1}\left(g z_{n+1}, F(z, x, y)\right)\right] \\
& \leqslant s\left(1+k_{1}\right)\left[d_{1}\left(g x_{n}, g x\right)+d_{1}\left(g y_{n}, g y\right)+d_{1}\left(g z_{n}, g z\right)\right]+s\left(1+k_{2}\right) k^{n} \delta_{0} \\
&+s k_{3}\left[d_{1}(g x, F(x, y, z))+d_{1}(g y, F(y, z, x))+d_{1}(g z, F(z, x, y))\right] .
\end{aligned}
$$

By taking $n \rightarrow \infty$ in the above inequality (2.18), we have

$$
\begin{aligned}
d_{1}(g x, F(x, y, z))+ & d_{1}(g y, F(y, z, x))+d_{1}(g z, F(z, x, y)) \\
& \leqslant s_{3}\left[d_{1}(g x, F(x, y, z))+d_{1}(g y, F(y, z, x))+d_{1}(g z, F(z, x, y))\right] .
\end{aligned}
$$

By the condition $0 \leqslant s k_{3}<1$ and (2.19), we can easily obtain that

$$
d_{1}(g x, F(x, y, z))+d_{1}(g y, F(y, z, x))+d_{1}(g z, F(z, x, y))=0,
$$

which implies that

$$
g x=F(x, y, z)), g y=F(y, z, x)), g z=F(z, x, y) .
$$


Therefore, we conclude that $(x, y, z)$ is the tripled coincidence point of $g$ and $F$.

Next, we show the uniqueness of the triple point of coincidence of $g$ and $F$. Assume that $\left(x^{*}, y^{*}, z^{*}\right)$ is another tripled coincidence point of mappings $g$ and F. By (2.1), we derive

$$
\begin{aligned}
d_{1}\left(g x, g x^{*}\right)+ & d_{1}\left(g y, g y^{*}\right)+d_{1}\left(g z, g z^{*}\right) \\
= & d_{1}\left(F(x, y, z), F\left(x^{*}, y^{*}, z^{*}\right)\right)+d_{1}\left(F(y, z, x), F\left(y^{*}, z^{*}, x^{*}\right)\right)+d_{1}\left(F(z, x, y), F\left(z^{*}, x^{*}, y^{*}\right)\right) \\
\leqslant & \left.k_{1}\left[d_{2}\left(g x, g x^{*}\right)+d_{2}\left(g y, g y^{*}\right)\right)+d_{2}\left(g z, g z^{*}\right)\right] \\
& +k_{2}\left[d_{2}(g x, F(x, y, z))+d_{2}(g y, F(y, z, x))+d_{2}(g z, F(z, x, y))\right] \\
& +k_{3}\left[d_{2}\left(g x^{*}, F\left(x^{*}, y^{*}, z^{*}\right)\right)+d_{2}\left(g y^{*}, F\left(y^{*}, z^{*}, x^{*}\right)\right)+d_{2}\left(g z^{*}, F\left(z^{*}, x^{*}, y^{*}\right)\right)\right] \\
= & k_{1}\left[d_{2}\left(g x, g x^{*}\right)+d_{2}\left(g y, g y^{*}\right)+d_{2}\left(g z, g z^{*}\right)\right] \\
\leqslant & k_{1}\left[d_{1}\left(g x, g x^{*}\right)+d_{1}\left(g y, g y^{*}\right)+d_{1}\left(g z, g z^{*}\right)\right] .
\end{aligned}
$$

By virtue of $0 \leqslant k_{1} \leqslant k_{1}+k_{2}+k_{3}<1$ and (2.20), we deduce

$$
d_{1}\left(g x, g x^{*}\right)+d_{1}\left(g y, g y^{*}\right)+d_{1}\left(g z, g z^{*}\right)=0 .
$$

This implies that $g x=g x^{*}, g y=g y^{*}$ and $g z=g z^{*}$. So that the triple point of coincidence of $g$ and $F$ is unique.

Next, we show that $g x=g y=g z$. In fact, it follows from (2.1) that

$$
\begin{aligned}
d_{1}(g x, g y)+ & d_{1}(g y, g z)+d_{1}(g z, g x) \\
= & d_{1}(F(x, y, z), F(y, z, x))+d_{1}(F(y, z, x), F(z, x, y))+d_{1}(F(z, x, y), F(x, y, z)) \\
\leqslant & k_{1}\left[d_{2}(g x, g y)+d_{2}(g y, g z)+d_{2}(g z, g x)\right] \\
& +k_{2}\left[d_{2}(g x, F(x, y, z))+d_{2}(g y, F(y, z, x))+d_{2}(g z, F(z, x, y))\right] \\
& +k_{3}\left[d_{2}(g y, F(y, z, x))+d_{2}(g z, F(z, x, y))+d_{2}(g x, F(x, y, z))\right] \\
= & k_{1}\left[d_{2}(g x, g y)+d_{2}(g y, g z)+d_{2}(g z, g x)\right] \\
\leqslant & k_{1}\left[d_{1}(g x, g y)+d_{1}(g y, g z)+d_{1}(g z, g x)\right] .
\end{aligned}
$$

By making use of $0 \leqslant k_{1} \leqslant k_{1}+k_{2}+k_{3}<1$ and (2.21), we deduce

$$
d_{1}(g x, g y)+d_{1}(g y, g z)+d_{1}(g z, g x)=0 .
$$

This means that $g x=g y=g z$.

Finally, if $g$ and $F$ are $w$-compatible, then we have $g(F(x, y, z))=F(g x, g y, g z)$. Therefore, by taking $u=g x$, we have $u=g x=F(x, y, z)=g y=F(y, z, x)=g z=F(z, x, y)$, hence we have

$$
g u=g g x=g(F(x, y, z))=F(g x, g y, g z)=F(u, u, u) .
$$

Thus, $(g u, g u, g u)$ is a coupled point of coincidence of $g$ and $F$, and by its uniqueness, we get $g u=g x$. Thus, we obtain $u=g u=F(u, u, u)$. Therefore, $(u, u, u)$ is the unique common tripled fixed point of $g$ and F. This completes the proof of Theorem 2.1.

In Theorem 2.1, if we take $d_{1}(x, y)=d_{2}(x, y)=d(x, y)$ for all $x, y \in X$, then we get the following corollary.

Corollary 2.2. Let $(\mathrm{X}, \mathrm{d})$ be a rectangular b-metrics space with coefficient $\mathrm{s} \geqslant 1$, and $\mathrm{F}: \mathrm{X} \times \mathrm{X} \times \mathrm{X} \rightarrow \mathrm{X}$ and $\mathrm{g}: \mathrm{X} \rightarrow \mathrm{X}$ be two mappings. Suppose that there exist $\mathrm{k}_{1}, \mathrm{k}_{2}$ and $\mathrm{k}_{3}$ in $[0,1)$ with $0 \leqslant \mathrm{k}_{1}+\mathrm{k}_{2}+\mathrm{k}_{3}<1$ and $0 \leqslant s k_{3}<1$ such that the condition

$$
\begin{gathered}
d(F(x, y, z), F(u, v, w))+d(F(y, z, x), F(v, w, u))+d(F(z, x, y), F(w, u, v)) \\
\leqslant k_{1}[d(g x, g u)+d(g y, g v)+d(g z, g w)]
\end{gathered}
$$




$$
\begin{aligned}
& +k_{2}[d(g x, F(x, y, z))+d(g y, F(y, z, x))+d(g z, F(z, x, y))] \\
& +k_{3}[d(g u, F(u, v, w))+d(g v, F(v, w, u))+d(g w, F(w, u, v))]
\end{aligned}
$$

holds for all $(x, y, z),(u, v, w) \in X \times X \times X$.

If $\mathrm{F}(\mathrm{X} \times \mathrm{X} \times \mathrm{X}) \subseteq \mathrm{g}(\mathrm{X})$ and $\mathrm{g}(\mathrm{X})$ is complete, then $\mathrm{g}$ and $\mathrm{F}$ have a tripled coincidence point $(x, y, z) \in$ $X \times X \times X$, satisfying that $g x=F(x, y, z)=g y=F(y, z, x)=g z=F(z, x, y)$.

Moreover, if $\mathrm{g}$ and $\mathrm{F}$ are $\mathrm{w}$-compatible, then $\mathrm{g}$ and $\mathrm{F}$ have a unique common tripled fixed point of the form $(\mathfrak{u}, \mathrm{u}, \mathrm{u})$, which satisfies that $\mathrm{u}=\mathrm{gu}=\mathrm{F}(\mathrm{u}, \mathrm{u}, \mathrm{u})$.

Corollary 2.3. Let $\mathrm{d}_{1}$ and $\mathrm{d}_{2}$ be two rectangular b-metrics on $\mathrm{X}$ such that $\mathrm{d}_{2}(\mathrm{x}, \mathrm{y}) \leqslant \mathrm{d}_{1}(\mathrm{x}, \mathrm{y})$ for all $\mathrm{x}, \mathrm{y} \in \mathrm{X}$, $\left(\mathrm{X}, \mathrm{d}_{1}\right)$ with coefficient $\mathrm{s} \geqslant 1$, and $\mathrm{F}: \mathrm{X} \times \mathrm{X} \times \mathrm{X} \rightarrow \mathrm{X}$ and $\mathrm{g}: \mathrm{X} \rightarrow \mathrm{X}$ be two mappings. Suppose that there exist $a_{i} \in[0,1)(i=1,2,3, \cdots, 9)$ with $0 \leqslant a_{1}+a_{2}+a_{3}+\cdots+a_{9}<1$ and $0 \leqslant s\left(a_{7}+a_{8}+a_{9}\right)<1$ such that the condition

$$
\begin{aligned}
d_{1}(F(x, y, z), F(u, v, w)) \leqslant & a_{1} d_{2}(g x, g u)+a_{2} d_{2}(g y, g v)+a_{3} d_{2}(g z, g w) \\
& +a_{4} d_{2}(g x, F(x, y, z))+a_{5} d_{2}(g y, F(y, z, x))+a_{6} d_{2}(g z, F(z, x, y)) \\
& +a_{7} d_{2}(g u, F(u, v, w))+a_{8} d_{2}(g v, F(v, w, u))+a_{9} d_{2}(g w, F(w, u, v)),
\end{aligned}
$$

holds for all $(x, y, z),(u, v, w) \in X \times X \times X$.

If $\mathrm{F}(\mathrm{X} \times \mathrm{X} \times \mathrm{X}) \subseteq \mathrm{g}(\mathrm{X})$ and $\mathrm{g}(\mathrm{X})$ is $\mathrm{d}_{1}$-complete, then $\mathrm{g}$ and $\mathrm{F}$ have a tripled coincidence point $(x, y, z) \in$ $\mathrm{X} \times \mathrm{X} \times \mathrm{X}$, which satisfies that $\mathrm{gx}=\mathrm{F}(\mathrm{x}, \mathrm{y}, z)=\mathrm{gy}=\mathrm{F}(\mathrm{y}, z, \mathrm{x})=\mathrm{g} z=\mathrm{F}(z, \mathrm{x}, \mathrm{y})$.

Moreover, if $\mathrm{g}$ and $\mathrm{F}$ are $w$-compatible, then $\mathrm{g}$ and $\mathrm{F}$ have a unique common tripled fixed point of the form $(\mathrm{u}, \mathrm{u}, \mathrm{u})$, which satisfies that $\mathrm{u}=\mathrm{gu}=\mathrm{F}(\mathrm{u}, \mathrm{u}, \mathrm{u})$.

Proof. Given $(x, y),(u, v),(z, w) \in X \times X$. It follows from (2.22) that

$$
\begin{aligned}
d_{1}(F(x, y, z), F(u, v, w)) \leqslant & a_{1} d_{2}(g x, g u)+a_{2} d_{2}(g y, g v)+a_{3} d_{2}(g z, g w) \\
& +a_{4} d_{2}(g x, F(x, y, z))+a_{5} d_{2}(g y, F(y, z, x))+a_{6} d_{2}(g z, F(z, x, y)) \\
& +a_{7} d_{2}(g u, F(u, v, w))+a_{8} d_{2}(g v, F(v, w, u))+a_{9} d_{2}(g w, F(w, u, v)), \\
d_{1}(F(y, z, x), F(v, w, u)) \leqslant & a_{1} d_{2}(g y, g v)+a_{2} d_{2}(g z, g w)+a_{3} d_{2}(g x, g u) \\
& +a_{4} d_{2}(g y, F(y, z, x))+a_{5} d_{2}(g z, F(z, x, y))+a_{6} d_{2}(g x, F(x, y, z)) \\
& +a_{7} d_{2}(g v, F(v, w, u))+a_{8} d_{2}(g w, F(w, u, v))+a_{9} d_{2}(g u, F(u, v, w)),
\end{aligned}
$$

and

$$
\begin{aligned}
d_{1}(F(z, x, y), F(w, u, v)) \leqslant & a_{1} d_{2}(g z, g w)+a_{2} d_{2}(g x, g u)+a_{3} d_{2}(g y, g v) \\
& +a_{4} d_{2}(g z, F(z, x, y))+a_{5} d_{2}(g x, F(x, y, z))+a_{6} d_{2}(g y, F(y, z, x)) \\
& +a_{7} d_{2}(g w, F(w, u, v))+a_{8} d_{2}(g u, F(u, v, w))+a_{9} d_{2}(g v, F(v, w, u))
\end{aligned}
$$

Combining (2.23), (2.24) and (2.25), we have

$$
\begin{aligned}
d_{1}(F(x, y, z), F(u, v, w))+ & d_{1}(F(y, z, x), F(v, w, u))+d_{1}(F(z, x, y), F(w, u, v)) \\
\leqslant & \left(a_{1}+a_{2}+a_{3}\right)\left[d_{2}(g x, g u)+d_{2}(g y, g v)+d_{2}(g z, g w)\right] \\
& +\left(a_{4}+a_{5}+a_{6}\right)\left[d_{2}(g x, F(x, y, z))+d_{2}(g y, F(y, z, x))+d_{2}(g z, F(z, x, y))\right] \\
+ & \left(a_{7}+a_{8}+a_{9}\right)\left[d_{2}(g u, F(u, v, w))+d_{2}(g v, F(v, w, u))+d_{2}(g w, F(w, u, v))\right] .
\end{aligned}
$$

Therefore, the result follows from Theorem 2.1.

Remark 2.4. If we take $d_{1}(x, y)=d_{2}(x, y)=d(x, y)$ for all $x, y \in X$, where $d$ is a rectangular b-metrics on $\mathrm{X}$, then Corollary 2.3 is reduced to a new result. 
The following corollary can be obtained from Theorem 2.1 immediately.

Corollary 2.5. Let $\mathrm{d}_{1}$ and $\mathrm{d}_{2}$ be two rectangular b-metrics on $X$ such that $\mathrm{d}_{2}(\mathrm{x}, \mathrm{y}) \leqslant \mathrm{d}_{1}(\mathrm{x}, \mathrm{y})$ for all $\mathrm{x}, \mathrm{y} \in \mathrm{X}$, and $\mathrm{F}: \mathrm{X} \times \mathrm{X} \times \mathrm{X} \rightarrow \mathrm{X}$ and $\mathrm{g}: \mathrm{X} \rightarrow \mathrm{X}$ be two mappings. Suppose that there exists $\mathrm{k} \in[0,1)$ such that the condition

$$
\begin{gathered}
d_{1}(F(x, y, z), F(u, v, w))+d_{1}(F(y, z, x), F(v, w, u))+d_{1}(F(z, x, y), F(w, u, v)) \\
\leqslant k\left[d_{2}(g x, g u)+d_{2}(g y, g v)+d_{2}(g z, g w)\right]
\end{gathered}
$$

holds for all $(x, y, z),(u, v, w) \in X \times X \times X$.

If $\mathrm{F}(\mathrm{X} \times \mathrm{X} \times \mathrm{X}) \subseteq \mathrm{g}(\mathrm{X})$ and $\mathrm{g}(\mathrm{X})$ is $\mathrm{d}_{1}$-complete, then $\mathrm{g}$ and $\mathrm{F}$ have a tripled coincidence point $(\mathrm{x}, \mathrm{y}, z) \in$ $\mathrm{X} \times \mathrm{X} \times \mathrm{X}$, which satisfies that $\mathrm{gx}=\mathrm{F}(\mathrm{x}, \mathrm{y}, z)=\mathrm{gy}=\mathrm{F}(\mathrm{y}, z, \mathrm{x})=\mathrm{g} z=\mathrm{F}(z, \mathrm{x}, \mathrm{y})$.

Moreover, if $\mathrm{g}$ and $\mathrm{F}$ are $\mathrm{w}$-compatible, then $\mathrm{g}$ and $\mathrm{F}$ have a unique common tripled fixed point of the form $(\mathfrak{u}, \mathrm{u}, \mathrm{u})$, which satisfies that $\mathrm{u}=\mathrm{gu}=\mathrm{F}(\mathrm{u}, \mathrm{u}, \mathrm{u})$.

Corollary 2.6. Let $\mathrm{d}_{1}$ and $\mathrm{d}_{2}$ be two rectangular b-metrics on $\mathrm{X}$ such that $\mathrm{d}_{2}(\mathrm{x}, \mathrm{y}) \leqslant \mathrm{d}_{1}(\mathrm{x}, \mathrm{y})$ for all $\mathrm{x}, \mathrm{y} \in \mathrm{X}$, and $\mathrm{F}: \mathrm{X} \times \mathrm{X} \times \mathrm{X} \rightarrow \mathrm{X}$ and $\mathrm{g}: \mathrm{X} \rightarrow \mathrm{X}$ be two mappings. Suppose that there exists $\mathrm{k} \in[0,1)$ such that the condition

$$
\begin{gathered}
d_{1}(F(x, y, z), F(u, v, w))+d_{1}(F(y, z, x), F(v, w, u))+d_{1}(F(z, x, y), F(w, u, v)) \\
\leqslant k\left[d_{2}(g x, F(x, y, z))+d_{2}(g y, F(y, z, x))+d_{2}(g z, F(z, x, y))\right]
\end{gathered}
$$

holds for all $(x, y, z),(u, v, w) \in X \times X \times X$.

If $\mathrm{F}(\mathrm{X} \times \mathrm{X} \times \mathrm{X}) \subseteq \mathrm{g}(\mathrm{X})$ and $\mathrm{g}(\mathrm{X})$ is $\mathrm{d}_{1}$-complete, then $\mathrm{g}$ and $\mathrm{F}$ have a tripled coincidence point $(x, y, z) \in$ $X \times X \times X$, which satisfies that $g x=F(x, y, z)=g y=F(y, z, x)=g z=F(z, x, y)$.

Moreover, if $\mathrm{g}$ and $\mathrm{F}$ are $\mathrm{w}$-compatible, then $\mathrm{g}$ and $\mathrm{F}$ have a unique common tripled fixed point of the form $(\mathrm{u}, \mathrm{u}, \mathrm{u})$, which satisfies that $\mathrm{u}=\mathrm{gu}=\mathrm{F}(\mathrm{u}, \mathrm{u}, \mathrm{u})$.

Corollary 2.7. Let $\mathrm{d}_{1}$ and $\mathrm{d}_{2}$ be two rectangular b-metrics on $\mathrm{X}$ such that $\mathrm{d}_{2}(\mathrm{x}, \mathrm{y}) \leqslant \mathrm{d}_{1}(\mathrm{x}, \mathrm{y})$ for all $\mathrm{x}, \mathrm{y} \in \mathrm{X}$, $\left(\mathrm{X}, \mathrm{d}_{1}\right)$ with coefficient $\mathrm{s} \geqslant 1$, and $\mathrm{F}: \mathrm{X} \times \mathrm{X} \times \mathrm{X} \rightarrow \mathrm{X}$ and $\mathrm{g}: \mathrm{X} \rightarrow \mathrm{X}$ be two mappings. Suppose that there exists $k \in[0,1)$ with $0 \leqslant s k<1$ such that the condition

$$
\begin{gathered}
d_{1}(F(x, y, z), F(u, v, w))+d_{1}(F(y, z, x), F(v, w, u))+d_{1}(F(z, x, y), F(w, u, v)) \\
\leqslant k\left[d_{2}(g u, F(u, v, w))+d_{2}(g v, F(v, w, u))+d_{2}(g w, F(w, u, v))\right]
\end{gathered}
$$

holds for all $(x, y, z),(u, v, w) \in X \times X \times X$.

If $\mathrm{F}(\mathrm{X} \times \mathrm{X} \times \mathrm{X}) \subseteq \mathrm{g}(\mathrm{X})$ and $\mathrm{g}(\mathrm{X})$ is $\mathrm{d}_{1}$-complete, then $\mathrm{g}$ and $\mathrm{F}$ have a tripled coincidence point $(x, y, z) \in$ $X \times X \times X$, which satisfies that $\mathrm{gx}=\mathrm{F}(\mathrm{x}, \mathrm{y}, z)=\mathrm{gy}=\mathrm{F}(\mathrm{y}, z, \mathrm{x})=\mathrm{g} z=\mathrm{F}(z, x, y)$.

Moreover, if $\mathrm{g}$ and $\mathrm{F}$ are $\mathrm{w}$-compatible, then $\mathrm{g}$ and $\mathrm{F}$ have a unique common tripled fixed point of the form $(\mathrm{u}, \mathrm{u}, \mathrm{u})$, which satisfies that $\mathrm{u}=\mathrm{gu}=\mathrm{F}(\mathrm{u}, \mathrm{u}, \mathrm{u})$.

Let $\mathrm{g}=\mathrm{I}_{\mathrm{X}}$ (the identity mapping) in Theorem 2.1 and Corollaries 2.2, 2.3, 2.5-2.7. Then we have the following results.

Corollary 2.8. Let $\mathrm{d}_{1}$ and $\mathrm{d}_{2}$ be two rectangular b-metrics on $\mathrm{X}$ such that $\mathrm{d}_{2}(x, y) \leqslant \mathrm{d}_{1}(\mathrm{x}, \mathrm{y})$ for all $\mathrm{x}, \mathrm{y} \in \mathrm{X}$, $\left(X, \mathrm{~d}_{1}\right)$ with coefficient $\mathrm{s} \geqslant 1$ and $\mathrm{F}: \mathrm{X} \times \mathrm{X} \times \mathrm{X} \rightarrow \mathrm{X}$ be a mapping. Suppose that there exist $\mathrm{k}_{1}, \mathrm{k}_{2}$ and $\mathrm{k}_{3}$ in $[0,1)$ with $0 \leqslant k_{1}+k_{2}+k_{3}<1$ and $0 \leqslant s k_{3}<1$ such that the condition

$$
\begin{aligned}
& d_{1}(F(x, y, z), F(u, v, w))+d_{1}(F(y, z, x), F(v, w, u))+d_{1}(F(z, x, y), F(w, u, v)) \\
& \leqslant k_{1}\left[d_{2}(x, u)+d_{2}(y, v)+d_{2}(z, w)\right]+k_{2}\left[d_{2}(x, F(x, y, z))+d_{2}(y, F(y, z, x))\right. \\
& \left.+d_{2}(z, F(z, x, y))\right]+k_{3}\left[d_{2}(u, F(u, v, w))+d_{2}(v, F(v, w, u))+d_{2}(w, F(w, u, v))\right]
\end{aligned}
$$

holds for all $(x, y, z),(u, v, w) \in X \times X \times X$.

If $\mathrm{X}$ is $\mathrm{d}_{1}$-complete, then $\mathrm{F}$ has a unique tripled fixed point of the form $(\mathrm{u}, \mathrm{u}, \mathrm{u})$, which satisfies that $\mathrm{u}=$ $F(u, u, u)$. 
Corollary 2.9. Let $(\mathrm{X}, \mathrm{d})$ be a complete rectangular b-metrics space with coefficient $\mathrm{s} \geqslant 1$, and $\mathrm{F}: \mathrm{X} \times \mathrm{X} \times \mathrm{X} \rightarrow \mathrm{X}$ be a mapping. Suppose that there exist $\mathrm{k}_{1}, \mathrm{k}_{2}$ and $\mathrm{k}_{3}$ in $[0,1)$ with $0 \leqslant \mathrm{k}_{1}+\mathrm{k}_{2}+\mathrm{k}_{3}<1$ and $0 \leqslant s \mathrm{k}_{3}<1$ such that the condition

$$
\begin{aligned}
& d(F(x, y, z), F(u, v, w))+d(F(y, z, x), F(v, w, u))+d(F(z, x, y), F(w, u, v)) \\
& \leqslant k_{1}[d(x, u)+d(y, v)+d(z, w)]+k_{2}[d(x, F(x, y, z))+d(y, F(y, z, x))+d(z, F(z, x, y))] \\
& +k_{3}[d(u, F(u, v, w))+d(v, F(v, w, u))+d(w, F(w, u, v))]
\end{aligned}
$$

holds for all $(x, y, z),(u, v, w) \in X \times X \times X$.

Then $\mathrm{F}$ has a unique tripled fixed point of the form $(\mathrm{u}, \mathrm{u}, \mathrm{u})$, which satisfies that $\mathrm{u}=\mathrm{gu}=\mathrm{F}(\mathrm{u}, \mathrm{u}, \mathrm{u})$.

Corollary 2.10. Let $d_{1}$ and $d_{2}$ be two rectangular b-metrics on $X$ such that $d_{2}(x, y) \leqslant d_{1}(x, y)$ for all $x, y \in X$, $\left(\mathrm{X}, \mathrm{d}_{1}\right)$ with coefficient $\mathrm{s} \geqslant 1$ and $\mathrm{F}: \mathrm{X} \times \mathrm{X} \times \mathrm{X} \rightarrow \mathrm{X}$ be a mapping. Suppose that there exist $\mathrm{a}_{\mathrm{i}} \in[0,1)$ $(i=1,2,3, \cdots, 9)$ with $0 \leqslant a_{1}+a_{2}+a_{3}+\cdots+a_{9}<1$ and $0 \leqslant s\left(a_{7}+a_{8}+a_{9}\right)<1$ such that the condition

$$
\begin{aligned}
d_{1}(F(x, y, z), F(u, v, w)) \leqslant & a_{1} d_{2}(x, u)+a_{2} d_{2}(y, v)+a_{3} d_{2}(z, w) \\
& +a_{4} d_{2}(x, F(x, y, z))+a_{5} d_{2}(y, F(y, z, x))+a_{6} d_{2}(z, F(z, x, y)) \\
& +a_{7} d_{2}(u, F(u, v, w))+a_{8} d_{2}(v, F(v, w, u))+a_{9} d_{2}(w, F(w, u, v)),
\end{aligned}
$$

holds for all $(x, y, z),(u, v, w) \in X \times X \times X$.

If $\left(\mathrm{X}, \mathrm{d}_{1}\right)$ is complete, then $\mathrm{F}$ has a unique tripled fixed point of the form $(\mathrm{u}, \mathrm{u}, \mathrm{u})$, which satisfies that $\mathrm{u}=\mathrm{gu}=$ $\mathrm{F}(\mathrm{u}, \mathrm{u}, \mathrm{u})$.

Corollary 2.11. Let $\mathrm{d}_{1}$ and $\mathrm{d}_{2}$ be two rectangular b-metrics on $\mathrm{X}$ such that $\mathrm{d}_{2}(\mathrm{x}, \mathrm{y}) \leqslant \mathrm{d}_{1}(\mathrm{x}, \mathrm{y})$ for all $\mathrm{x}, \mathrm{y} \in \mathrm{X}$, and $\mathrm{F}: \mathrm{X} \times \mathrm{X} \times \mathrm{X} \rightarrow \mathrm{X}$ be a mapping. Suppose that there exists $\mathrm{k} \in[0,1)$ such that the condition

$$
\begin{gathered}
d_{1}(F(x, y, z), F(u, v, w))+d_{1}(F(y, z, x), F(v, w, u))+d_{1}(F(z, x, y), F(w, u, v)) \\
\leqslant k\left[d_{2}(x, u)+d_{2}(y, v)+d_{2}(z, w)\right]
\end{gathered}
$$

holds for all $(x, y, z),(u, v, w) \in X \times X \times X$.

If $\left(X, d_{1}\right)$ is complete, then $F$ has a unique tripled fixed point of the form $(\mathrm{u}, \mathrm{u}, \mathrm{u})$, which satisfies that $\mathrm{u}=\mathrm{gu}=$ $\mathrm{F}(\mathrm{u}, \mathrm{u}, \mathrm{u})$.

Corollary 2.12. Let $\mathrm{d}_{1}$ and $\mathrm{d}_{2}$ be two rectangular b-metrics on $\mathrm{X}$ such that $\mathrm{d}_{2}(\mathrm{x}, \mathrm{y}) \leqslant \mathrm{d}_{1}(\mathrm{x}, \mathrm{y})$ for all $\mathrm{x}, \mathrm{y} \in \mathrm{X}$, and $\mathrm{F}: \mathrm{X} \times \mathrm{X} \times \mathrm{X} \rightarrow \mathrm{X}$ be a mapping. Suppose that there exists $\mathrm{k} \in[0,1)$ such that the condition

$$
\begin{gathered}
d_{1}(F(x, y, z), F(u, v, w))+d_{1}(F(y, z, x), F(v, w, u))+d_{1}(F(z, x, y), F(w, u, v)) \\
\leqslant k\left[d_{2}(x, F(x, y, z))+d_{2}(y, F(y, z, x))+d_{2}(z, F(z, x, y))\right]
\end{gathered}
$$

holds for all $(x, y, z),(u, v, w) \in X \times X \times X$.

If $\left(X, \mathrm{~d}_{1}\right)$ is complete, then $\mathrm{F}$ has a unique tripled fixed point of the form $(\mathrm{u}, \mathrm{u}, \mathrm{u})$, which satisfies that $\mathrm{u}=\mathrm{gu}=$ $\mathrm{F}(\mathrm{u}, \mathrm{u}, \mathrm{u})$.

Corollary 2.13. Let $d_{1}$ and $d_{2}$ be two rectangular b-metrics on $X$ such that $d_{2}(x, y) \leqslant d_{1}(x, y)$ for all $x, y \in X$, $\left(X, \mathrm{~d}_{1}\right)$ with coefficient $\mathrm{s} \geqslant 1$ and $\mathrm{F}: \mathrm{X} \times \mathrm{X} \times \mathrm{X} \rightarrow \mathrm{X}$ and $\mathrm{g}: \mathrm{X} \rightarrow \mathrm{X}$ be two mappings. Suppose that there exists $k \in[0,1)$ with $0 \leqslant s k<1$ such that the condition

$$
\begin{gathered}
d_{1}(F(x, y, z), F(u, v, w))+d_{1}(F(y, z, x), F(v, w, u))+d_{1}(F(z, x, y), F(w, u, v)) \\
\leqslant k\left[d_{2}(u, F(u, v, w))+d_{2}(v, F(v, w, u))+d_{2}(w, F(w, u, v))\right]
\end{gathered}
$$

holds for all $(x, y, z),(u, v, w) \in X \times X \times X$.

If $\left(\mathrm{X}, \mathrm{d}_{1}\right)$ is complete, then $\mathrm{F}$ has a unique tripled fixed point of the form $(\mathrm{u}, \mathrm{u}, \mathrm{u})$, which satisfies that $\mathrm{u}=\mathrm{gu}=$ $\mathrm{F}(\mathrm{u}, \mathrm{u}, \mathrm{u})$.

Remark 2.14. If we take coefficient $s=1$ (for the space X) in Theorem 2.1 and Corollaries 2.2, 2.3, 2.5-2.13, then several new results can be obtain in two rectangular metric space. 


\section{Application to integral equations}

Example 3.1. Let $X=\mathbb{R}$ and define $d: X \times X \rightarrow \mathbb{R}^{+}$as $d(x, y)=|x-y|^{k}, x, y \in X$, where $k \geqslant 1$. Then $(X, d)$ be a rectangular b-metric space with coefficient $s=3^{k-1}$.

In fact, obviously conditions (RbM1) and (RbM2) in Definition 1.3 are satisfied. Now we show that condition (RbM3) holds for $\mathrm{k}$. By using the following inequality

$$
(a+b+c)^{k} \leqslant 3^{k-1}\left(a^{k}+b^{k}+c^{k}\right), \forall a, b, c \in \mathbb{R}^{+} \text {and } k \geqslant 1,
$$

we can get

$$
\begin{aligned}
\mathrm{d}(x, y) & =|x-y|^{k}=|(x-z)+(z-w)+(w-y)|^{k} \\
& \leqslant(|x-z|+|z-w|+|w-y|)^{k} \\
& \leqslant 3^{k-1}\left(|x-z|^{k}+|z-w|^{k}+|w-y|^{k}\right) \\
& =3^{k-1}(d(x, z)+d(z, w)+d(w, y)),
\end{aligned}
$$

for all $x, y, z, w \in X$. This means that (RbM3) holds, hence $(X, d)$ is a rectangular b-metric space.

Example 3.2. Let $X=\mathbb{R}$ and $d_{1}, d_{2}$ are two rectangular b-metrics in $X$ such that

$$
d_{1}(x, y)=(x-y)^{2}, \quad d_{2}(x, y)=\frac{(x-y)^{2}}{4}, \forall x, y \in X
$$

Define $\mathrm{F}: \mathrm{X} \times \mathrm{X} \times \mathrm{X} \rightarrow \mathrm{X}$ and $\mathrm{g}: \mathrm{X} \rightarrow \mathrm{X}$ respectively by

$$
\mathrm{F}(x, y, z)=\frac{x-y+z}{3}, g x=4 x, \forall x, y, z \in X .
$$

It is easy to see that $g(X)$ is $d_{1}$-complete, and $F$ and $g$ are $\omega$-compatible.

On the other hand, we have

$$
\begin{aligned}
d_{1}(F(x, y, z), F(u, v, w)) & =(F(x, y, z)-F(u, v, w))^{2} \\
& =\left(\frac{x-y+z}{3}-\frac{u-v+w}{3}\right)^{2}=\left(\frac{x-u}{3}+\frac{v-y}{3}+\frac{z-w}{3}\right)^{2} \\
& \leqslant 3\left(\frac{(x-u)^{2}}{9}+\frac{(y-v)^{2}}{9}+\frac{(z-w)^{2}}{9}\right) \\
& =\frac{(x-u)^{2}}{3}+\frac{(y-v)^{2}}{3}+\frac{(z-w)^{2}}{3} \\
& =\frac{(4 x-4 u)^{2}}{48}+\frac{(4 y-4 v)^{2}}{48}+\frac{(4 z-4 w)^{2}}{48} \\
& =\frac{(g x-g u)^{2}}{48}+\frac{(g y-g v)^{2}}{48}+\frac{(g z-g w)^{2}}{48} \\
& =\frac{1}{12}\left[d_{2}(g x, g u)+d_{2}(g y, g v)+d_{2}(g z, g w)\right] .
\end{aligned}
$$

By similar arguments as above, we can show that

$$
d_{1}(F(y, z, x), F(v, w, u)) \leqslant \frac{1}{12}\left[d_{2}(g y, g v)+d_{2}(g z, g w)+d_{2}(g x, g u)\right],
$$

and

$$
d_{1}(F(z, x, y), F(w, u, v)) \leqslant \frac{1}{12}\left[d_{2}(g z, g w)+d_{2}(g x, g u)+d_{2}(g y, g v)\right] .
$$


Combining the above three inequalities, we obtain

$$
\begin{aligned}
d_{1}(F(x, y, z), F(u, v, w))+ & d_{1}(F(y, z, x), F(v, w, u))+d_{1}(F(z, x, y), F(w, u, v)) \\
& \leqslant \frac{1}{4}\left[d_{2}(g x, g u)+d_{2}(g y, g v)+d_{2}(g z, g w)\right] .
\end{aligned}
$$

Then by Corollary 2.5, $\mathrm{F}$ and $\mathrm{g}$ have a unique common tripled fixed point, in fact $(0,0,0)$ is the unique common tripled fixed point of mappings of $F$ and $g$.

Next, we assume that $X=C[a, b]$ is the set of all continuous functions. Define two rectangular $b$ metrics respectively by

$$
d_{1}(x, y)=\max _{t \in[a, b]}|x(t)-y(t)|^{k}, \quad d_{2}(x, y)=\frac{\max _{t \in[a, b]}|x(t)-y(t)|^{k}}{3}, \quad \forall x, y \in X, \quad(k \geqslant 1) .
$$

Then the coefficient of two rectangular b-metrics is $s=3^{k-1}$. Consider the nonlinear integral equation set as follows

$$
\left\{\begin{array}{l}
x(r)=K(r)+\int_{a}^{b} G(r, t)[f(r, x(t))+g(r, y(t))+h(r, z(t))] d t, \\
y(r)=K(r)+\int_{a}^{b} G(r, t)[f(r, y(t))+g(r, z(t))+h(r, x(t))] d t, \\
z(r)=K(r)+\int_{a}^{b} G(r, t)[f(r, z(t))+g(r, x(t))+h(r, y(t))] d t .
\end{array}\right.
$$

Now, we will analyze (3.1) under the following conditions:

(i) $f, g, h:[a, b] \times X \rightarrow \mathbb{R}$ are three continuous functions.

(ii) $\mathrm{K}:[\mathrm{a}, \mathrm{b}] \rightarrow \mathbb{R}$ are continuous functions.

(iii) $G:[a, b] \times[a, b] \rightarrow \mathbb{R}^{+}$is a continuous function.

(iv) There exist $L_{i}>0(i=1,2,3)$ such that for all $x, y \in X$,

$$
\left\{\begin{array}{l}
|f(r, x(t))-f(r, y(t))| \leqslant L_{1}|x-y| \\
|g(r, x(t))-g(r, y(t))| \leqslant L_{2}|x-y| \\
|h(r, x(t))-h(r, y(t))| \leqslant L_{3}|x-y|
\end{array}\right.
$$

(v)

$$
\max _{r \in[a, b]}\left(\int_{a}^{b} G(r, t) d t\right)^{k}<\frac{1}{3^{2 k+1} L^{k}}
$$

where $L=\max \left\{L_{1}, L_{2}, L_{3}\right\}$.

Theorem 3.3. Under the conditions (i)-(v), the integral equation (3.1) has a unique common solution on $[\mathrm{a}, \mathrm{b}]$.

Proof. Define $\mathrm{F}: \mathrm{X}^{3} \rightarrow \mathrm{X}$ and $\mathrm{g}: \mathrm{X} \rightarrow \mathrm{X}$ respectively by

$$
\begin{aligned}
F(x, y, z)(r) & =K(r)+\int_{a}^{b} G(r, t)[f(r, x(t))+g(r, y(t))+h(r, z(t))] d t, \quad \forall x, y, z \in X, \quad r \in[a, b] \\
g x & =x, \quad \forall x \in X .
\end{aligned}
$$


By Example 3.1, (iv) and (v), we have

$$
\begin{aligned}
& |\mathrm{F}(x, y, z)(\mathrm{r})-\mathrm{F}(\mathrm{u}, v, w)(\mathrm{r})|^{\mathrm{k}} \\
& =\mid \int_{a}^{b} G(r, t)[f(r, x(t))-f(r, u(t))] d t+\int_{a}^{b} G(r, t)[g(r, y(t))-g(r, v(t))] d t \\
& +\left.\int_{a}^{b} G(r, t)[h(r, z(t))-h(r, w(t))] d t\right|^{k} \\
& \leqslant 3^{k-1}\left[\left.|| \int_{a}^{b} G(r, t)[f(r, x(t))-f(r, u(t))] d t\right|^{k}+\left|\int_{a}^{b} G(r, t)[g(r, y(t))-g(r, v(t))] d t\right|^{k}\right. \\
& \left.+\left|\int_{a}^{b} G(r, t)[h(t, z(t))-h(t, w(t))] d t\right|^{k}\right] \\
& \leqslant 3^{k-1}\left[L_{1}^{k}\left(\max _{t \in[a, b]}|x(t)-u(t)|\right)^{k}+L_{2}^{k}\left(\max _{t \in[a, b]}|y(t)-v(t)|\right)^{k}\right. \\
& \left.+\mathrm{L}_{3}^{\mathrm{k}}\left(\max _{\mathrm{t} \in[\mathrm{a}, \mathrm{b}]}|z(\mathrm{t})-w(\mathrm{t})|\right)^{\mathrm{k}}\right]\left(\int_{\mathrm{a}}^{\mathrm{b}} \mathrm{G}(\mathrm{r}, \mathrm{t}) \mathrm{dt}\right)^{\mathrm{k}} \\
& \leqslant 3^{k-1} L^{k}\left[\max _{t \in[a, b]}|x(t)-u(t)|^{k}+\max _{t \in[a, b]}|y(t)-v(t)|^{k}+\max _{t \in[a, b]}|z(t)-w(t)|^{k}\right]\left(\int_{a}^{b} G(r, t) d t\right)^{k} \\
& =3^{k-1} L^{k}\left[d_{1}(x, u)+d_{1}(y, v)+d_{1}(z, w)\right]\left(\int_{a}^{b} G(r, t) d t\right)^{k} \\
& \leqslant 3^{k-1} L^{k}\left[d_{1}(x, u)+d_{1}(y, v)+d_{1}(z, w)\right] \cdot \frac{1}{3^{2 k+1} L^{k}} \\
& \leqslant \frac{1}{3^{k+2}}\left[d_{1}(x, u)+d_{1}(y, v)+d_{1}(z, w)\right]
\end{aligned}
$$

It follows from the above inequality that

$$
\begin{aligned}
d_{1}(F(x, y, z), F(u, v, w)) & =\max _{r \in[a, b]}|F(x, y, z)(r)-F(u, v, w)(r)|^{k} \\
& \leqslant \frac{1}{3^{k+2}}\left[d_{1}(x, u)+d_{1}(y, v)+d_{1}(z, w)\right] .
\end{aligned}
$$

By similar arguments as above,

$$
\begin{aligned}
& d_{1}(F(y, z, x), F(v, w, u)) \leqslant \frac{1}{3^{k+2}}\left[d_{1}(y, v)+d_{1}(z, w)+d_{1}(x, u)\right] \\
& d_{1}(F(z, x, y), F(w, u, v)) \leqslant \frac{1}{3^{k+2}}\left[d_{1}(z, w)+d_{1}(x, u)+d_{1}(y, v)\right]
\end{aligned}
$$

It follows from (3.2), (3.3) and (3.4) that

$$
\begin{aligned}
d_{1}(F(x, y, z), F(u, v, w))+ & d_{1}(F(y, z, x), F(v, w, u))+d_{1}(F(z, x, y), F(w, u, v)) \\
& \leqslant \frac{1}{3^{k+1}}\left[d_{1}(x, u)+d_{1}(y, v)+d_{1}(z, w)\right]=\frac{1}{3^{k}}\left[d_{2}(x, u)+d_{2}(y, v)+d_{2}(z, w)\right] .
\end{aligned}
$$

By Corollary 2.5 we assert that there exists $\tau \in X$ such that $F(\tau, \tau, \tau)=g \tau=\tau$ which implies that $\tau$ is the unique solution of equation set (3.1). 
Example 3.4. We assume that $X=C[0,1]$ is the set of all continuous functions defined on $[0,1]$. Define $\left(X, d_{1}\right)$ and $\left(X, d_{2}\right)$ respectively by

$$
d_{1}(x, y)=\max _{r \in[0,1]}|x(r)-y(r)|^{2}, \quad d_{2}(x, y)=\max _{r \in[0,1]} \frac{|x(r)-y(r)|^{2}}{3}, \forall x, y \in X .
$$

Then the coefficient of two rectangular b-metrics is $k=2$. Consider the following nonlinear integral equation set:

$$
\left\{\begin{array}{l}
x(r)=2^{r}+\int_{0}^{1} \frac{\sin (r \cdot \pi)}{3 \sqrt{3}+t}\left[\frac{e^{-r x(t)}}{3}+\frac{\sin (y(t) \cdot \pi)}{6 \pi+r}+\frac{\cos (r)}{4} \cdot \frac{|z(t)|}{1+|z(t)|}\right] d t, \\
y(r)=2^{r}+\int_{0}^{1} \frac{\sin (r \cdot \pi)}{3 \sqrt{3}+t}\left[\frac{e^{-r y(t)}}{3}+\frac{\sin (z(t) \cdot \pi)}{6 \pi+r}+\frac{\cos (r)}{4} \cdot \frac{|x(t)|}{1+|x(t)|}\right] d t, \\
z(r)=2^{r}+\int_{0}^{1} \frac{\sin (r \cdot \pi)}{3 \sqrt{3}+t}\left[\frac{e^{-r z(t)}}{3}+\frac{\sin (x(t) \cdot \pi)}{6 \pi+r}+\frac{\cos (r)}{4} \cdot \frac{|y(t)|}{1+|y(t)|}\right] d t .
\end{array}\right.
$$

Define the function $K:[0,1] \rightarrow \mathbb{R}, G:[0,1] \times[0,1] \rightarrow \mathbb{R}^{+}, f, g, h:[0,1] \times X \rightarrow \mathbb{R}$ respectively by: $K(r)=2^{r}$, $G(r, t)=\frac{\sin (r \cdot \pi)}{3 \sqrt{3}+t}, f(r, x(t))=\frac{e^{-r x(t)}}{3}, g(r, x(t))=\frac{\sin (x(t) \cdot \pi)}{6 \pi+r}, h(r, x(t))=\frac{\cos (r)}{4} \cdot \frac{|x(t)|}{1+|x(t)|}$.

We can easily obtain that $K(t), G(r, t), f(r, x), g(r, x), h(r, x)$ are continuous functions. Also, for all $r \in[0,1]$ and $x, y \in X$, we have

$$
\begin{aligned}
|f(r, x)-f(r, y)| & =\left|\frac{e^{-r x}}{3}-\frac{e^{-r y}}{3}\right|=\left|\frac{-r e^{-r \xi}}{3}(x-y)\right| \leqslant \frac{1}{3}|x-y|, \\
|g(r, x)-g(r, y)| & =\left|\frac{\sin (x \cdot \pi)}{6 \pi+r}-\frac{\sin (y \cdot \pi)}{6 \pi+r}\right| \leqslant \frac{|\sin (x \cdot \pi)-\sin (y \cdot \pi)|}{6 \pi} \\
& =\frac{1}{6 \pi}|\pi \cdot \cos (\eta \cdot \pi) \cdot(x-y)| \leqslant \frac{1}{6}|x-y|, \\
|h(r, x)-h(r, y)| & =\left|\frac{\cos (r)}{4} \cdot \frac{|x|}{1+|x|}-\frac{\cos (r)}{4} \cdot \frac{|y|}{1+|y|}\right| \leqslant \frac{1}{4}\left|\frac{|x|}{1+|x|}-\frac{|y|}{1+|y|}\right| \\
& \leqslant \frac{1}{4}\left|\frac{1+|x|-1}{1+|x|}-\frac{1+|y|-1}{1+|y|}\right| \leqslant \frac{1}{4}\left|\frac{1}{1+|y|}-\frac{1}{1+|x|}\right| \\
& =\frac{1}{4}\left|\frac{1}{(1+\zeta)^{2}}(|x|-|y|)\right| \leqslant \frac{1}{4}|| x|-| y|| \leqslant \frac{1}{4}|x-y| .
\end{aligned}
$$

Here $r \in[0,1], \mathrm{L}_{1}=\frac{1}{3}, \mathrm{~L}_{2}=\frac{1}{6}, \mathrm{~L}_{3}=\frac{1}{4}, \mathrm{~L}=\max \left\{\mathrm{L}_{1}, \mathrm{~L}_{2}, \mathrm{~L}_{3}\right\}=\frac{1}{3}, \xi, \eta$ exist between $\mathrm{x}$ and $\mathrm{y}, \zeta$ exist between $|x|$ and $|y|$.

$$
\max _{r \in[0,1]}\left(\int_{0}^{1} G(r, t) d t\right)^{2}=\max _{r \in[0,1]}\left(\int_{0}^{1} \frac{\sin (r \cdot \pi)}{3 \sqrt{3}+t} d t\right)^{2} \leqslant\left(\frac{1}{3 \sqrt{3}}\right)^{2}=\frac{1}{3^{3}}=\frac{1}{3^{2 k+1} L^{k}} .
$$

Consequently, all the conditions of Theorem 3.3 are satisfied. Hence the integral equation set (3.5) has the unique solution in $\mathrm{C}[0,1]$.

\section{Acknowledgment}

This work is supported by the National Natural Science Foundation of China $(11071169,11271105)$, the Natural Science Foundation of Zhejiang Province (Y6110287).

\section{References}

[1] T. Abdeljawad, D. Türkog̃lu, Locally convex valued rectangular metric spaces and the Kannan's fixed point theorem, J. Comput. Anal. Appl., 14 (2012), 484-494. 1

[2] J. Ahmad, M. Arshad, C. Vetro, On a theorem of Khan in a generalized metric space, Int. J. Anal., 2013 (2013), 6 pages.

[3] M. Arshad, J. Ahmad, E. Karapınar, Some common fixed point results in rectangular metric spaces, Int. J. Anal., 2013 (2013), 7 pages. 1 
[4] H. Aydi, M. Abbas, W. Sintunavarat, P. Kumam, Tripled fixed point of W-compatible mappings in abstract metric spaces, Fixed Point Theory Appl., 2012 (2012), 20 pages. 1.7, 1.8, 1.9

[5] H. Aydi, A. Felhi, S. Sahmim, Common fixed points in rectangular b-metric spaces using (E.A) property, J. Adv. Math. Stud., 8 (2015), 159-169. 1

[6] H. Aydi, E. Karapınar, H. Lakzian, Fixed point results on a class of generalized metric spaces, Math. Sci. (Springer), 2012 (2012), 6 pages. 1

[7] N. Bilgili, E. Karapınar, D. Turkoglu, A note on common fixed points for $(\psi, \alpha, \beta)$-weakly contractive mappings in generalized metric spaces, Fixed Point Theory Appl., 2013 (2013), 6 pages. 1

[8] A. Branciari, A fixed point theorem of Banach-Caccioppoli type on a class of generalized metric spaces, Publ. Math. Debrecen, 57 (2000), 31-37. 1, 1.2

[9] N. Cakić, Coincidence and common fixed point theorems for $(\psi, \phi)$ weakly contractive mappings in generalized metric spaces, Filomat, 27 (2013), 1415-1423. 1

[10] S. Czerwik, Contraction mappings in b-metric spaces, Acta Math. Inform. Univ. Ostraviensis, 1 (1993), 5-11. 1, 1.1

[11] P. Das, L. K. Dey, Fixed point of contractive mappings in generalized metric spaces, Math. Slovaca, 59 (2009), 499-504. 1

[12] C. Di Bari, P. Vetro, Common fixed points in generalized metric spaces, Appl. Math. Comput., 218 (2012), 7322-7325. 1

[13] H.-S. Ding, M. Imdad, S. Radenović, J. Vujaković, On some fixed point results in b-metric, rectangular and b-rectangular metric spaces, Arab J. Math. Sci., 22 (2016), 151-164. 1

[14] H.-S. Ding, V. Ozturk, S. Radenović, On some new fixed point results in b-rectangular metric spaces, J. Nonlinear Sci. Appl., 8 (2015), 378-386. 1.4, 1

[15] İ. M. Erhan, E. Karapınar, T. Sekulić, Fixed points of $(\psi, \phi)$ contractions on rectangular metric spaces, Fixed Point Theory Appl., 2012 (2012), 12 pages. 1

[16] A. Flora, A. Bellour, A. Al-Bsoul, Some results in fixed point theory concerning generalized metric spaces, Mat. Vesnik, 61 (2009), 203-208. 1

[17] R. George, S. Radenović, K. P. Reshma, S. Shukla, Rectangular b-metric space and contraction principles, J. Nonlinear Sci. Appl., 8 (2015), 1005-1013. 1, 1.3, 1.4, 1.5

[18] R. George, R. Rajagopalan, Common fixed point results for $\psi-\phi$ contractions in rectangular metric spaces, Bull. Math. Anal. Appl., 5 (2013), 44-52. 1

[19] H. Işik, D. Türkog̃lu, Common fixed points for $(\psi, \alpha, \beta)$-weakly contractive mappings in generalized metric spaces, Fixed Point Theory Appl., 2013 (2013), 6 pages.

[20] W. A. Kirk, N. Shahzad, Generalized metrics and Caristi's theorem, Fixed Point Theory Appl., 2013 (2013), 9 pages.

[21] B. K. Lahiri, P. Das, Fixed point of a Ljubomir Ćirić's quasi-contraction mapping in a generalized metric space, Publ. Math. Debrecen, 61 (2002), 589-594.

[22] H. Lakzian, B. Samet, Fixed points for $(\psi, \phi)$-weakly contractive mappings in generalized metric spaces, Appl. Math. Lett., 25 (2012), 902-906.

[23] V. La Rosa, P. Vetro, Common fixed points for $\alpha-\psi-\phi$-contractions in generalized metric spaces, Nonlinear Anal. Model. Control, 19 (2014), 43-54.

[24] S. K. Malhotra, S. Shukla, R. Sen, Some fixed point theorems for ordered Reich type contractions in cone rectangular metric spaces, Acta Math. Univ. Comenian. (N.S.), 82 (2013), 165-175.

[25] S. Moradi, D. Alimohammadi, New extensions of Kannan fixed-point theorem on complete metric and generalized metric spaces, Int. J. Math. Anal. (Ruse), 5 (2011), 2313-2320. 1

[26] J. R. Roshan, V. Parvaneh, Z. Kadelburg, N. Hussain, New fixed point results in b-rectangular metric spaces, Nonlinear Anal. Model. Control, 21 (2016), 614-634. 1, 1.3

[27] B. Samet, A fixed point theorem in a generalized metric space for mappings satisfying a contractive condition of integral type, Int. J. Math. Anal. (Ruse), 3 (2009), 1265-1271. 1

[28] B. Samet, Discussion on "A fixed point theorem of Banach-Caccioppoli type on a class of generalized metric spaces" by A. Branciari [MR1771669], Publ. Math. Debrecen, 76 (2010), 493-494. 1

[29] B. Samet, C. Vetro, Coupled fixed point, F-invariant set and fixed point of N-order, Ann. Funct. Anal., 1 (2010), 46-56. 1.6

[30] S. Shukla, G-(F, $\tau)$-contractions in partial rectangular metric spaces endowed with a graph and fixed point theorems, TWMS J. Appl. Eng. Math., 6 (2016), 342-353. 1 